\title{
Keyless Abdominal Rope-lifting Surgery (KARS)
}

\section{Kahraman Ülker*}

Department of Obstetrics and Gynecology, Kafkas University School of Medicine, Kars, Turkey

\begin{abstract}
Keyless Rope-lifting Surgery (KARS) is a novel, single incision, gasless laparoscopic surgery technique. Various operations including adnexal cyst excision, oophorectomy, diagnostic laparoscopy, tubal ligation, hysterectomy and cholecystectomy may be performed through the intra-umbilical transverse incision without using $\mathrm{CO}_{2}$ pneumoperitoneum and thus the trocars (keyless). In KARS the abdominal cavity is accessed through the $1.5-2 \mathrm{~cm}$ intra-umbilical transverse incision. The anterior abdominal wall is elevated by the sutures loaded into the Veress cannula. The elevation creates room for the operations. All operations are performed through the intra-umbilical incision by using laparoscopic and/ or conventional hand instruments. Here in this paper, the KARS techniques and the preliminary results of the specific gynecological procedures are presented.
\end{abstract}

Keywords: Abdominal; $\mathrm{CO}_{2}$; Gynecology and obstetrics; Minimal access surgical procedures; Pneumoperitoneum; Single-port; Surgical (technical); Technical (endoscopy)

\section{Introduction}

Laparoscopic surgery has gained a worldwide popularity for the last two decades. Its popularity probably depends on the experience of less pain and fewer complications in comparison with laparotomy. However, as an intra-abdominal procedure it still has the same potential of intra-operative and postoperative risks of laparotomy [1-4].

A smaller incision resulting in less post-operative scarring, less pain and medication for pain, reduced exposure of internal organs to possible external contaminants, reduced bleeding and the chance of needing a blood transfusion, fewer complications, and earlier discharge and work are the advantageous of laparoscopic surgery. However, it also has some risks and disadvantageous such as Veress needle or trocar injuries to blood vessels or bowels, unseen electrical burns leading organ perforation, hypothermia and peritoneal trauma due to increased exposure to cold and dry gases during insufflation, intolerability of pneumoperitoneum in patients with cardiac and pulmonary diseases, and the increase of anesthesia risks by pneumoperitoneum and shoulder pain [5-9].

Researchers have conducted and published numerous trails in order to minimize the side effects and maximize the advantages of laparoscopic surgery [10-15]. Keyless Abdominal Rope-lifting Surgery (KARS) is one of the laparoscopic surgery techniques claiming to improve the outcomes of laparoscopic surgery [16,17]. The aim of this paper is to define the major aspects of this gasless, single incision abdominal access technique and present our experiences in various operations performed by using the technique.

\section{Surgical Technique}

\section{Preoperative preparation}

As in every laparoscopic and major intra-abdominal surgery bowel preparation is crucial in order to maintain an optimum vision of the intra-abdominal operative field. After three days of preoperative feeding containing largely liquids the patients are treated with a mixture of 2.4 $\mathrm{g} / 5 \mathrm{ml}$ monobasic sodium phosphate and $0.9 \mathrm{~g} / 5 \mathrm{ml}$ bibasic sodium phosphate per twice daily with a dose of $45 \mathrm{ml}$, each time. Oral intake is prohibited at $11 \mathrm{p} . \mathrm{m}$. before the operation day and a mixture of $19 \mathrm{~g}$ sodium dihydrogen phosphate and $7 \mathrm{~g}$ disodium hydrogen phosphate in $135 \mathrm{ml}$ solution is used rectally at 6 a.m. on the operation day.

\section{Operative theatre preparations}

As in conventional $\mathrm{CO}_{2}$ laparoscopy the patients are prepared in the supine decubitus position on the operative table. Abdominal cleansing and sterile draping is as usual, however, special care has to be given to the cleaning of the umbilical region. Mechanical and chemical cleaning of the umbilical region with sterile gauze on the tip of a fine instrument is mandatory in order to prevent wound infection.

A universal ether screen at the level of the line between the anteriorsuperior iliac crests is maintained $10-12 \mathrm{~cm}$ above the abdominal surface and covered with sterile drapes.

General anesthesia is preferred due to the frequent necessity of the elevation of the operative region of the body. However, regional anesthesia is also an option for minor and short procedures (e.g. tubal sterilization and salpingo oophorectomy/oophorectomy).

\section{Abdominal access}

The construction of the access pathway into the abdominal cavity in KARS is similar to open laparoscopic technique. The skin covering the umbilical fold is lifted with 2 clamps bilaterally and a third clamp elevates the center of the umbilicus. Depending on the necessity of multiple instrument use a $1.5-2 \mathrm{~cm}$ transverse incision is performed at the center of the umbilicus (Figure 1). The subcutaneous tissue is dissected bluntly by using the tip of a fine instrument such as a Kelly clamp. After the identification of the underlying fascia, two strong, fine clamps are used to fixate and elevate the fascia. The facial layer is dissected with a fine dissection scissor and two fine retractors are used to apply a moderate stretching force in order to bluntly widen the opening.

\section{Rope-lifting process}

In order to guide the rest of the process the abdominal entry site is lifted first. The lower and upper border of the underlying fascia is sutured with a number 0 or 1 delayed-absorbable suture at 6 and 12 o'clock positions, respectively (Figure 2). The inner side of the abdominal wall is examined digitally for possible adhesions. The entry

*Corresponding author: Kahraman Ülker, Department of Obstetrics and Gynecology, Kafkas University School of Medicine, Kars, Turkey, E-mail kahramanulker@hotmail.com

Received December 15, 2012; Accepted January 25, 2013; Published February 05, 2013

Citation: Ülker K (2013) Keyless Abdominal Rope-lifting Surgery (KARS). Surgery S12: 007. doi:10.4172/2161-1076.S12-007

Copyright: @ 2013 Ülker K. This is an open-access article distributed under the terms of the Creative Commons Attribution License, which permits unrestricted use, distribution, and reproduction in any medium, provided the original author and source are credited. 


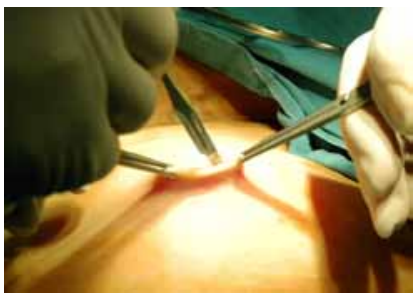

Figure 1: A $1.5-2 \mathrm{~cm}$ transverse incision is performed at the center of the umbilicus in order to create the intra-abdominal access pathway.

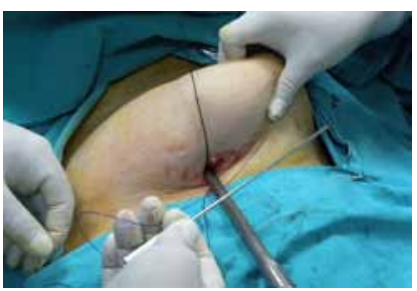

Figure 2: The lower and upper border of the fascia underlying the umbilical incision is sutured with a number 0 or 1 delayed-absorbable suture at 6 and 12 o'clock positions, respectively.

site is elevated with the help of the entry site sutures and the telescope is introduced gently and slowly into the incision. The abdominal viscera are observed for possible injuries or adhesions.

The Veress needle is unloaded from its cannula and one tip of a \#1 nylon suture is inserted into the cannula (Figure 3). The loaded cannula is introduced into the elevated entry site. The tip of the cannula is inserted towards the abdominal wall $1-2 \mathrm{~cm}$ below the entry under direct vision. The cannula is then slid just above the peritoneum or rectus sheet and maintained in an upward direction to avoid bowel injury.

The cannula is oriented laterally right $6-7 \mathrm{~cm}$ to avoid injury of the epigastric vessels and the abdominal wall is pierced from the inside towards the outside at approximately $5 \mathrm{~cm}$ below the entry. The tip of the suture is unloaded from the cannula outside the abdominal wall and the unloaded cannula is removed back from the entry. The second tip of the suture is inserted into the cannula and the same steps are repeated. However, the tip of the cannula is taken outside the abdominal cavity $5 \mathrm{~cm}$ below the first tip.

After the same steps are repeated at the contra-lateral side (the tip of the loaded cannula is oriented to the left side) of the abdominal wall the sutures are ready to lift the abdominal wall. An assistant elevates the abdominal wall and the surgeon ties the sutures over the pre-prepared sterile ether screen or retractor (Figure 4).

The abdominal cavity is visualized with the introduced telescope. In order to prevent the staining of the optic of the telescope it is better to stretch the entry site sutures during telescope introduction. If the provided intra-abdominal space does not reach the desired volumes, additional lifting sutures can be placed to the sites where further elevations are required. Small protruding areas (e.g. protrusion of the peritoneal folds or adipose tissue which enclosed the operative field, particularly in the over-weighted patient) can be lifted by using a suture just penetrating the skin. The skin suture is also tied to over the same ether screen or retractor. Following the creation of the intra-abdominal operative space, various operations can be performed by using the single incision access route. The procedure does not require the creation of pneumoperitoneum, thus the laparoscopic hand instruments are used without their trocars (keyless).

\section{Wound closure}

At the end of the surgery the lifting sutures are simply cut and removed. The facial layer is closed with a number 0 delayed-absorbable suture. The lifting facial sutures facilitate the closure. The umbilical skin is closed with 3-0 or 4-0 intra-cutaneous absorbable sutures and hidden into the umbilical fold. The anterior abdominal wall including the umbilical incision and the scars created by the passage of the Veress cannula is cleaned with an antiseptic solution. The umbilical incision is covered with sterile gauze or an adhesive plaster.

\section{Operations Performed by Using Kars}

We have been using the KARS technique since the first half of 2010. Up to date operations including diagnostic laparoscopy and tubal patency testing for infertility work up, ovarian cystectomy, oophorectomy, salpingectomy, salpingostomy, tubal milking, tubal sterilization, lysis of intra-abdominal adhesions and hysterectomy were performed. Although the preliminary results of all specific surgical procedures were presented in various congress and meetings [18-23], only the results of cystectomies were published in medical journals $[16,17]$.

From August 2012 on, cholecystectomies were performed by using KARS and the first two cases were presented in the international congress of the European Society of Surgery held in November 2012 in İstanbul, Turkey [24]. The operation times for the 63 and 40 year old patients were 42 and 110 minutes, respectively.

Table 1 summarizes the characteristics of the patients and perioperative findings of various gynecological surgeries $(n=84)$ performed by using KARS until October 2012. All operations were performed by using KARS without conversion to conventional laparoscopy or laparotomy.

Most of the included women were operated electively and none of them had malignant diseases. However, two women with ruptured

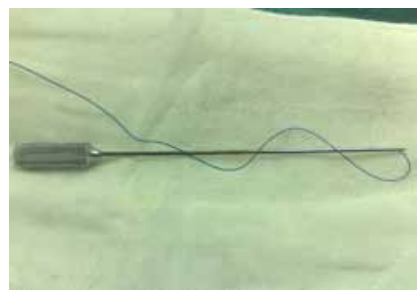

Figure 3: The Veress needle is unloaded from its cannula and one tip of a \#1 nylon suture is inserted into the cannula.

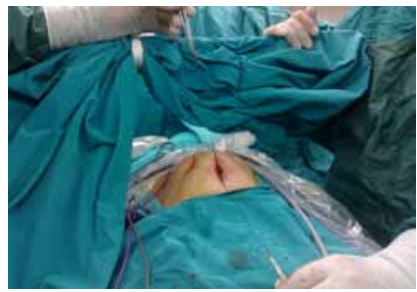

Figure 4: Final view after the completion of the rope-lifting process. 


\begin{tabular}{|c|c|c|c|c|c|c|c|}
\hline$N=84$ & Age & BMI $\left(\mathbf{k g} / \mathrm{m}^{2}\right)$ & $\mathrm{AAT}^{4}(\mathrm{~min})$ & TOT $^{5}(\mathrm{~min})$ & Hct $^{6}$ drop & $\mathrm{Hb}^{7}$ drop & Hospital stay \\
\hline Adnexal cysts ${ }^{1}(n=27)$ & $37.00 \pm 1.24$ & $26.09 \pm 5.00$ & $21.70 \pm 5.24$ & $92.67 \pm 31.64$ & $2,66 \pm 1,59$ & $0.96 \pm 0.52$ & $1.59 \pm 0.50$ \\
\hline Hysterectomy $^{2}(n=8)$ & $48.87 \pm 2.64$ & $28.38 \pm 3.79$ & $22.75 \pm 4.68$ & $165.2 \pm 31.67$ & $3.29 \pm 1.57$ & $1.05 \pm 0.47$ & $2.00 \pm 0.92$ \\
\hline Ectopic pregnancy $^{3}(n=6)$ & $32.00 \pm 7.18$ & $26.95 \pm 4.37$ & $19.33 \pm 7.55$ & $82.33 \pm 31.42$ & $2.17 \pm 1.89$ & $0.67 \pm 0.39$ & $1.83 \pm 0.41$ \\
\hline Tubal ligation $(n=37)$ & $36.81 \pm 3.81$ & $26.32 \pm 4.51$ & $15.81 \pm 6.47$ & $29.11 \pm 12.07$ & $2.88 \pm 1.67$ & $0.92 \pm 0.54$ & $0.40 \pm 0.50$ \\
\hline Diagnostic laparoscopy + Tubal patency testing $(n=6)$ & $30.00 \pm 5.83$ & $26.28 \pm 4.65$ & $19.17 \pm 4.71$ & $39.50 \pm 12.40$ & $2.35 \pm 1.06$ & $0.82 \pm 0.35$ & $0.33 \pm 0.52$ \\
\hline
\end{tabular}

${ }^{1}$ Oophorectomy was performed for the post menopausal women; ${ }^{2}$ Hysterectomies included bilateral salpingo-oophorectomy in postmenopausal women; ${ }^{3}$ Managemen of ectopic pregnancy included salpingectomy, salpingostomy or tubal milking depending on the clinical situation; ${ }^{4}$ AAT: Abdominal access time, time needed for the construction of lifting ropes; ${ }^{5}$ TOT: Total operative time, time needed for the total operation beginning from skin incision and ending with the closure of the incision; ${ }^{6} \mathrm{Hct}$ Hematocrit level change ${ }^{7} \mathrm{Hb}$ : Hemoglobin

Table 1: Selected characteristics of some gynecological operations performed by Keyless Rope-Lifting Surgery (KARS). The results are presented as mean \pm standard deviation.

tubal pregnancies and intra-abdominal bleeding, and a woman with the torsion of an ovarian cyst were operated in emergency conditions.

Abnormal uterine bleeding and myoma uteri were the indications for hysterectomy operations. The removal of the uterus and the closure of the vaginal opening were performed trans-vaginally. In all other operations the tissues were retrieved through the umbilical incision.

We did not observe any intra-operative complication other than minor bleedings which occurred during hysterectomy, salpingostomi and tubal milking. The bleedings did not necessitate blood transfusion in any patient.

In our previous study the preliminary results showed less postoperative pain scores after KARS operations [19], however our study group included KARS and single incision laparoscopic surgery (SILS) operations. In addition, either the study group (KARS and SILS) or the control groups (multiport $\mathrm{CO}_{2}$ laparoscopy and laparotomy) included various operations. Although our studies comparing the results of specific operations by using KARS, SILS, multiport laparoscopy and laparotomy are underway, preliminary results seem better pain scores in KARS. In addition, none of the 84 women operated by using KARS experienced shoulder pain.

Simple oral analgesics following the postoperative immediate 50 $\mathrm{mg}$ meperidine I.M. were adequate for postoperative pain relief.

In the post-operative period, the umbilical skin was kept covered with the sterile gauze for two days and then uncovered. The patients were allowed to take a shower, however they were ordered to keep the umbilical area clean and dry. The four prick scars created by the Veress cannula were left open and we did not order any special medication for them.

The recovery of intra umbilical transvers incision of the KARS procedure was generally completed in 7 to 12 days. All patients were examined at the post-operative $7^{\text {th }}$ day. Out of first 26 patients eight patients had hyperemia and bruising on umbilical skin. The hyperemia resolved in four to five days and the bruising dissolved in six to eight days. We did not observe these findings after using fine clamps for lifting the umbilical skin prior to the transvers incision. Three patients experienced intermittent serous discharge from the umbilical incision that lasted 10 to 12 days. Most of our patients were living in the rural area and working in the agricultural sector. Most of them returned their routine working practice on the $20^{\text {th }}$ day post-operatively. Except the aforementioned minor side effects we did not observe any major or minor complication related to KARS procedure.

During KARS procedures a variety of conventional surgical and laparoscopic instruments alongside a telescope could be introduced through the same single incision. Because of the gasless feature of the procedure the trocars were not used to prevent gas leakage. Thus, the technique was called keyless abdominal rope-lifting surgery: KARS. In addition, the trocar-less use of the hand instruments provided more space and enabled the use of multiple instruments through the same single incision. Moreover, the trocar-less entry provided the freedom of using instruments that were not adapted specifically to the trocars. This feature enabled the use of some conventional surgical instruments. For example, an excised uterine tube or an ovary was easily removed by using a long Kocher clamp.

Various instruments including mono and bipolar cautery, vessel sealing devices and harmonic scalpels were used for tissue excision. Intra and extra corporeal knots were applied for tissue approximations, hemostasis or tubal ligations. However, the single incision nature of the procedure hardened the manipulation of the instruments.

\section{Discussion}

Keyless abdominal rope-lifting surgery (KARS) is a single incision, gasless laparoscopic technique which enables the operation of various surgical procedures through an intra-umbilical $1.5-2 \mathrm{~cm}$ incision.

\section{Strengths of KARS}

Because KARS is a modified open laparoscopy technique it minimizes the chance of visceral injuries which occur after the blind introduction of the Veress needle or trocars into the abdominal cavity [9].

Gasless nature of KARS avoids pneumoperitoneum associated side effects like hypercapnia, acidosis, gas embolism, pneumothorax, subcutaneous emphysema, deep venous thrombosis, instability of the hemo-dynamics, decrease in renal functions, and peritoneal oxidative stress [5-7,10,11,25-27]. Although the operative time and patient position limit the use of regional (spinal and epidural) anesthesia in KARS, gasless surgery is also more optimal for regional anesthesia.

In $\mathrm{CO}_{2}$ laparoscopy, in order to increase the intra-abdominal pressure, the viscera should be closed to the extra abdominal space. In addition, the trocars are needed to prevent gas leakage while inserting and using the hand instruments [28]. In contrast, during a KARS procedure the abdominal wall is elevated with surgical ropes and the intra-abdominal high pressure gas is useless. Thus the trocars are also useless and the trocar-less entry spares more space for additional instrument introduction. In addition, the instruments need to fit with just the incision which also allows the use of suitable conventional surgical instruments as well.

Aspiration of the $\mathrm{CO}_{2}$ used in conventional laparoscopy lowers the intra-abdominal pressure resulting in poorer vision and, in some instances, stains the optic of the telescope [28]. Irrigation and aspiration can be used freely in gasless procedures.

Additional ports used in conventional $\mathrm{CO}_{2}$ laparoscopy increase 
the post-operative pain rate, diminish cosmesis, and carry a risk of bleeding, hernia formation, or organ damage. Although, a single special port or a multichannel device is used in single incision laparoscopic surgeries, they have limited numbers of access holes; one for the telescope and two for hand instruments $[15,28,29]$. KARS has all of the advantages of the single incision surgery and permits the use of multiple instruments at the same time.

The facial layer of the port sites are not sutured in many instances if $10 \mathrm{~mm}$ trocars are used. Almost all facial layers are not sutured if $5 \mathrm{~mm}$ trocars are used and the unsutured facial openings increase the chance of hernia formation. In KARS the single incision at the facial layer is prepared for closure at the initial stage of the surgery and stitched easily as in usual open surgery.

\section{Limitations of KARS}

KARS has the same common disadvantages of all single incision laparoscopic surgeries such as the sword fighting of the instruments and the telescope, and the obstruction of the vision of the operative field by a hand instrument passing in front of the telescope. In single incision laparoscopic surgeries the hand instruments and the telescope are introduced parallel with each other into the abdominal cavity. In contrast, the hand instruments and the telescope are introduced into the abdominal cavity with some angulations. The parallel introduction of the instruments during single incision laparoscopic surgeries makes the manipulation of the instruments and the tissues more difficult, particularly during procedures requiring intra-corporal suturing. Likewise, manipulation of the instruments and tissues is more difficult in KARS procedures. For example, we needed 15 to 20 minutes for the placement of a Meltzer sliding knot during tubal ligations and most of the time we could not succeed at the first attempt.

Although stretching the ropes sutured to the facial layer of the entry site increases the diameter of the entry, the telescope is stained more frequently due to the trocar-less entry. Thus, a gentle and careful introduction of the telescope is mandatory in KARS.

\section{Comparison of KARS with the previous surgical techniques}

In KARS the features of laparoscopy and laparotomy are modified and united. Thus the surgeons should be familiar with both techniques. However, the abdominal access technique and the elevation procedure are simple and can be performed by any surgeon familiar to laparotomy.

Gasless (isobaric) laparoscopic surgery after elevating the abdominal wall with a lifting device $[30,31]$ has been used to perform operations including myomectomy [32-34], hysterectomy [35], ovarian cyst resection [36], colposuspension [37] and radical hysterectomy [38]. Various surgical instruments, such as a special device with an abdominal retractor, a subcutaneous lifting device or an airlift balloon retractor have been used in these procedures. In addition, there were multiple port sites in previous techniques and the trocars were also used. In KARS, we elevated the abdominal wall with the usual conventional surgical sutures and used the laparoscopic hand instruments without their trocars through a single port.

Laparoendoscopic single-site surgery (LESS) has gained world-wide popularity and various surgical procedures including gynecological cancer staging, salpingo-oophorectomy, ovarian cystectomy, laparoscopy assisted vaginal hysterectomy and laparoscopic total hysterectomy have been performed by using LESS [39-44]. In LESS procedures, two (or more) conventional ports or a single multichannel device (which enables the passage of instruments and optics) are used through a single incision. However, limited numbers of channels

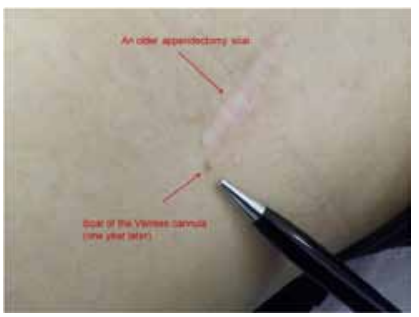

Figure 5: One year after a KARS operation the skin scar produced by the passage of the Veress cannula is compared with an older appendectomy scar.

allows for limited numbers of instruments to be used. In contrast KARS provides more space within a similar sized incision and enables the use of conventional surgical instruments.

KARS may have superiority in cosmesis (Figure 5) and fertility preservation in younger women caring about future fertility by allowing the enucleation of the capsules of some cysts outside the abdominal cavity [17]. Following the aspiration of the cyst content most of the adnexal cysts can be brought outside the abdominal cavity through the umbilical incision. Enucleation of the capsule of the cyst is easier outside the abdominal cavity. In addition, it is easier to dry the bleedings and repair the damaged tissue. Moreover, oriented pinpoint coagulation decreases the unnecessary ovarian tissue damage. In contrast, during $\mathrm{CO}_{2}$ laparoscopy (single incision or multiport) oozing blood obscures the real origin of the bleeding and blind coagulation causes extended tissue damage.

Although KARS has most of the advantageous features of laparoscopic surgery, our previous published study [17], our unpublished study comparing the tubal sterilizations performed by KARS and conventional $\mathrm{CO}_{2}$ laparoscopy (under review) and the cases presented in this paper suggest that the total operative time is long. Like the other single incision laparoscopic surgery techniques KARS increases the total operative time.

Although we present quite a lot cases in this paper, in order to achieve more definitive conclusions about the advantageous and disadvantageous features of KARS prospective randomized trials comparing KARS with the previous techniques are needed.

In conclusion as a gasless, single incision laparoscopy technique, KARS seems safe and effective in terms of cosmesis, less postoperative pain and fertility preservation. It allows the use of laparoscopic and conventional surgical instruments, and does not depend on trocars or special port devices. However, further comparative studies are needed to evaluate the feasibility of the procedure in specific operations.

\section{References}

1. Vagenas K, Spyrakopoulos P, Karanikolas M, Sakelaropoulos G, Maroulis I, et al. (2006) Mini-laparotomy cholecystectomy versus laparoscopic cholecystectomy: which way to go? Surg Laparosc Endosc Percutan Tech 16: 321-324.

2. Joris JL, Hinque VL, Laurent PE, Desaive CJ, Lamy ML (1998) Pulmonary function and pain after gastroplasty performed via laparotomy or laparoscopy in morbidly obese patients. $\mathrm{Br} \mathrm{J}$ Anaesth $80: 283-288$.

3. Karabacak O, Tiras MB, Taner MZ, Guner H, Yildiz A, et al. (1997) Smal diameter versus conventional laparoscopy: a prospective, self-controlled study. Hum Reprod 12: 2399-2401.

4. Busacca M, Fedele L, Bianchi S, Candiani M, Agnoli B, et al. (1998) Surgica treatment of recurrent endometriosis: laparotomy versus laparoscopy. Hum Reprod 13: 2271-2274.

5. Casati A, Valentini G, Ferrari S, Senatore R, Zangrillo A, et al. (1997) 
Cardiorespiratory changes during gynaecological laparoscopy by abdominal wall elevation: comparison with carbon dioxide pneumoperitoneum. $\mathrm{Br} \mathrm{J}$ Anaesth 78: 51-54

6. Schulze S, Lyng KM, Bugge K, Perner A, Bendtsen A, et al. (1999) Cardiovascular and respiratory changes and convalescence in laparoscopic colonic surgery: comparison between carbon dioxide pneumoperitoneum and gasless laparoscopy. Arch Surg 134: 1112-1118.

7. Bouvy ND, Giuffrida MC, Tseng LN, Steyerberg EW, Marquet RL, et al. (1998) Effects of carbon dioxide pneumoperitoneum, air pneumoperitoneum, and gasless laparoscopy on body weight and tumor growth. Arch Surg. 133:652656

8. Chapron C, Pierre F, Harchaoui Y, Lacroix S, Béguin S, et al. (1999) Gastrointestinal injuries during gynaecological laparoscopy. Hum Reprod 14: 333-337.

9. Chapron C, Querleu D, Bruhat MA, Madelenat P, Fernandez H, et al. (1998) Surgical complications of diagnostic and operative gynaecological laparoscopy: a series of 29,966 cases. Hum Reprod 13: 867-872.

10. Koivusalo AM, Kellokumpu I, Scheinin M, Tikkanen I, Mäkisalo $\mathrm{H}$, et al. (1998) A comparison of gasless mechanical and conventional carbon dioxide pneumoperitoneum methods for laparoscopic cholecystectomy. Anesth Analg 86:153-158.

11. Bickel A, Arzomanov T, Ivry S, Zveibl F, Eitan A (2004) Reversal of adverse hemodynamic effects of pneumoperitoneum by pressure equilibration. Arch Surg 139: 1320-1325.

12. Cevrioglu AS, Yilmaz S, Koken T, Tokyol C, Yilmazer M, et al. (2004) Comparison of the effects of low intra-abdominal pressure and ischaemic preconditioning on the generation of oxidative stress markers and inflammatory cytokines during laparoscopy in rats. Hum Reprod 19: 2144-2151.

13. Koivusalo AM, Kellokumpu I, Lindgren L (1996) Gasless laparoscopic cholecystectomy: comparison of postoperative recovery with conventional technique. Br J Anaesth 77: 576-580.

14. Desai MM, Rao PP, Aron M, Pascal-Haber G, Desai MR, et al. (2002) Scarless single port transumbilical nephrectomy and pyeloplasty: first clinical report. BJU Int 101: 83-8.

15. Park S, Bergs RA, Eberhart R, Baker L, Fernandez R, et al. (2007) Trocarless instrumentation for laparoscopy: magnetic positioning of intra-abdomina camera and retractor. Ann Surg 245: 379-384.

16. Ülker K, Ersöz M, Hüseyinoglu H (2011) Management of a giant ovarian cyst by keyless abdominal rope-lifting surgery (KARS). Kafkas J Med Sci 1: 25-29

17. Ülker K, Hüseyinoglu H, Kiliç N (2012) Management of benign ovarian cysts by a novel, gasless, single-incision laparoscopic technique: keyless abdominal rope-lifting surgery (KARS). Surg Endosc 27: 189-198.

18. Ülker K, Kiliç K, Hüseyinoglu Ü, Temur I, Gül A. Preliminary results of a novel, gasless, single incision abdominal access technique: Keyless Abdominal Rope-Lifting Surgery (KARS). AAGL 5th international congress on minimally invasive gynecology. April 2011: 175-7, Istanbul, Turkey.

19. Hüseyinoglu Ü, Ülker K, Temur I, Sülü B, Ok T, Kiliç K. Preliminary results of postoperative early pain scores of different abdominal access techniques: A prospective controlled study AAGL 5th international congress on minimally invasive gynecology. April 2011: 119-20, Istanbul, Turkey.

20. Ülker K. Management of ectopic pregnancies by using a single incision, gasless laparoscopic technique. 5th Aegean Gynecological endoscopic symposium. April 2012: 31, Izmir, Turkey.

21. Ülker K, Gül A, Hüseyinoglu Ü. 5. Hysterectomies by using a single incision, gasless laparoscopic technique. 5th Aegean Gynecological endoscopic symposium. April 2012: 18, Izmir, Turkey.

22. Ülker K, Kiliç N, Hüseyinoglu Ü. Tubal ligation by using a single incision, gasless laparoscopic technique. 5th Aegean Gynecological endoscopic symposium. April 2012: 20-1, Izmir, Turkey.

23. Ülker K. Keyless Abdominal Rope-Lifting Surgery- (KARS). 87th OMICS Group International Conference and Exhibition on Surgery, Anesthesia and Trichology. Surgery Curr Res 2012; 2(5): 60; San Antonio, USA.

24. Sülü B, llingi ED, Altun H, Günerhan Y, KöksalN, Ülker K. Laparoscopic single incision cholecystectomy without gas; Keyless Abdominal Rope-lifting Surgery (KARS). 16th Annual Meeting of European Society of Surgery. November 2012, Istanbul, Turkey.
25. Bablekos GD, Michaelides SA, Roussou T, Charalabopoulos KA (2006) Changes in breathing control and mechanics after laparoscopic vs open cholecystectomy. Arch Surg 141: 16-22.

26. Koivusalo AM, Kellokumpu I, Ristkari S, Lindgren L (1997) Splanchnic and rena deterioration during and after laparoscopic cholecystectomy: a comparison of the carbon dioxide pneumoperitoneum and the abdominal wall lift method. Anesth Analg 85: 886-891.

27. de Souza AM, Wang CC, Chu CY, Lam PM, Rogers MS (2003) The effect of intra-abdominal pressure on the generation of 8-iso prostaglandin F2alpha during laparoscopy in rabbits. Hum Reprod 18: 2181-2188.

28. Mishra RK. Textbook of practical laparoscopic surgery. New Delhi: Jaypee Brothers Medical Publishers Ltd.; 2009.

29. Fader AN, Levinson KL, Gunderson CC, Winder AD, Escobar PF (2011) Laparoendoscopic single-site surgery in gynaecology: A new frontier in minimally invasive surgery. J Minim Access Surg 7:71-77.

30. Chin AK, Moll FH, McColl MB, Reich H (1993) Mechanical peritoneal retraction as a replacement for carbon dioxide pneumoperitoneum. J Am Assoc Gynecol Laparosc 1: 62-66.

31. Smith RS, Fry WR, Tsoi EK, Henderson VJ, Hirvela ER, et al. (1993) Gasless laparoscopy and conventional instruments. The next phase of minimally invasive surgery. Arch Surg 1280: 1102-1107.

32. Chang FH, Soong YK, Cheng PJ, Lee CL, Lai YM, et al. (1996) Laparoscopic myomectomy of large symptomatic leiomyoma using airlift gasless laparoscopy: a preliminary report. Hum Reprod. 11: 1427-1432.

33. Damiani A, Melgrati L, Marziali M, Sesti F (2003) Gasless laparoscopic myomectomy. Indications, surgical technique and advantages of a new procedure for removing uterine leiomyomas. J Reprod Med. 48: 792-798.

34. Sesti F, Melgrati L, Damiani A, Piccione E (2006) Isobaric (gasless) laparoscopic uterine myomectomy. An overview. Eur J Obstet Gynecol Reprod Biol. 129: 9-14.

35. Kruschinski D, Homburg S, Wockel A. Kapur A, Reich H (2004) Lift-laparoscopic total hysterectomy as a routine procedure. Surg Technol Int 13:147-156.

36. Tintara H, Choobun $\mathrm{T}$ (2004) Laparoscopic adnexectomy for benign tuboovarian disease using the abdominal wall lift: a comparison to laparotomy. Int $J$ Gynaecol Obstet 84:147-155.

37. Davila GW, Stanford E, Korn A. Prospective trial of gasless laparoscopic Burch colposuspension using conventional surgical instruments (2004) J Am Assoc Gynecol Laparosc 11: 197-203

38. Bojahr B, Lober R, Straube W, Kohler G (1996) Gasless laparoscopic-assisted radical vaginal hysterectomy with lymphadenectomy for cervical carcinoma. J Am Assoc Gynecol Laparosc 3: S4-S5.

39. Fader AN, Escobar PF (2009) Laparoendoscopic single-site surgery (LESS) in gynecologic oncology: Technique and initial report. Gynecol Oncol 114:157161

40. Fader AN, Rojas-Espaillat L, Ibeanu O, Grumbine F, Escobar PF (2010) Laparoendoscopic single-site surgery (LESS) in gynecology: A multiinstitutional evaluation. Am J Obstet Gynecol 203: 501.e1-6.

41. Lee YY, Kim TJ, Kim CJ, Kang H, Choi CH, et al. (2012) Single-port access laparoscopic-assisted vaginal hysterectomy: A novel method with a wound retractor and a glove. J Minim Invasive Gynecol 16:450-453.

42. Yim GW, Jung YW, Paek J, Lee SH, Kwon HY, et al. (2010) Transumbilical single-port access versus conventional total laparoscopic hysterectomy: Surgical outcomes. Am J Obstet Gynecol 203:26.e1-6.

43. Savaris RF, Cavazzola LT (2009) Ectopic pregnancy: Laparoendoscopic single-site surgery- laparoscopic surgery through a single cutaneous incision. Fertil Steril 92:1170-e5-e7

44. Yoon BS, Park H, Seong SJ, Park CT, Park SW, et al. (2010) Single-port laparoscopic salpingectomy for the surgical treatment of ectopic pregnancy. J Minim Invasive Gynecol 17: 26-29. 